\title{
Brief
}

\section{Giant pseudoaneurysm after proximal aortic surgery treated by means of redo axillary artery cannulation and use of an Endoclamp device}

\author{
Francois Dagenais, MD, Pierre Voisine, MD, and Patrick Mathieu, MD, Quebec City, Quebec, Canada
}

From the Department of Cardiac Surgery, Laval Hospital, Quebec City, Quebec, Canada.

Received for publication Oct 20, 2004; revisions received Nov 17, 2004; accepted for publication Nov 23, 2004.

Address for reprints: Francois Dagenais, MD, Department of Cardiac Surgery, Laval Hospital, 2725 chemin Sainte-Foy, SainteFoy, Québec, Canada, G1V 4G5 (E-mail: francois.dagenais@chg.ulaval.ca).

J Thorac Cardiovasc Surg 2005;130:208-9

$0022-5223 / \$ 30.00$

Copyright (c) 2005 by The American Association for Thoracic Surgery

doi:10.1016/j.jtcvs.2004.11.037
$\mathrm{P}$

seudoeurysm formation after proximal aortic surgery, although rare, might supervene. ${ }^{1}$ We herein report a giant pseudoaneurysm after a Bentall procedure and a hemiarch replacement treated by the use of a redo axillary cannulation and an Endoclamp device (Cardiovations, Somerville, NJ).

\section{Clinical Summary}

A 70-year-old woman underwent a composite valve graft performed with a 27-mm Freestyle root conduit (Medtronic, Inc, Minneapolis, Minn) and a hemiarch replacement during circulatory arrest for an aneurysm. Arterial cannulation was performed through the right axillary artery. Ten months after the operation, a routine chest computed tomogram showed a 10-cm pseudoaneurysm pushing against the sternum. Angiography localized the leak between the Freestyle root and the Dacron graft (Figure 1). The close proximity of the pseudoaneurysm to the sternum mandated institution of cardiopulmonary bypass (CPB) and profound hypothermia before sternal opening. In an attempt to avoid circulatory arrest, the decision was made to arrest the heart by means of inflation of an Endoclamp device within the Dacron graft. After achievement of general anesthesia with bilateral radial arterial monitoring, a transjugular pulmonary vent (Cardiovations) was inserted. The previously dissected right axillary artery was exposed. The old $8-\mathrm{mm}$ graft was identified, the axillary artery was mobilized, and a new 8-mm graft was sutured to the artery. A Y-branched arterial cannula (Cardiovations) was inserted within the $8-\mathrm{mm}$ graft (Figure $2, A$ ). A $21 \mathrm{~F}$ venous cannula inserted through the right femoral vein was positioned in the right atrium. Once CPB was initiated, the Endoclamp device was inserted through the Y port of the arterial cannula and positioned in the ascending aortic graft. The Endoclamp device was subsequently inflated, and cardioplegic solution was infused through the proximal port of the catheter. Simultaneously, the sternum was reopened, and the pseudoaneurysm was entered. The leak was identified, and a Dacron interposition graft was sutured between the Freestyle root and the previous ascending aortic graft (Figure 2, B). Total CPB and crossclamp times were 88 and 52 minutes, respectively. The patient had an uneventful postoperative course. At 6 months postoperatively, the patient is asymptomatic, and results of chest computed tomography are normal.

\section{Discussion}

Giant pseudoaneurysms after aortic surgery remain an uncommon complication. ${ }^{2}$ Treatment is difficult and requires a well-planned operative strategy. In the presence of a giant pseudoaneurysm closely related to the sternum, peripheral initiation of CPB with sternal opening during profound hypothermia is the technique most often advocated. Complications related to the prolonged CPB time, such as coagulopathy or pulmonary and renal dysfunctions, are often encountered. Moreover, the duration of CPB has been shown to be an independent predictor of perioperative death for patients undergoing reoperation of the aortic root or ascending aorta. ${ }^{3,4}$

The present case illustrates a huge pseudoaneurysm with sternal contact. CPB was initiated through the previous right axillary artery. Reuse of a dissected right axillary artery is unreported to our knowledge. This technique offers the advantages of antegrade perfusion, 

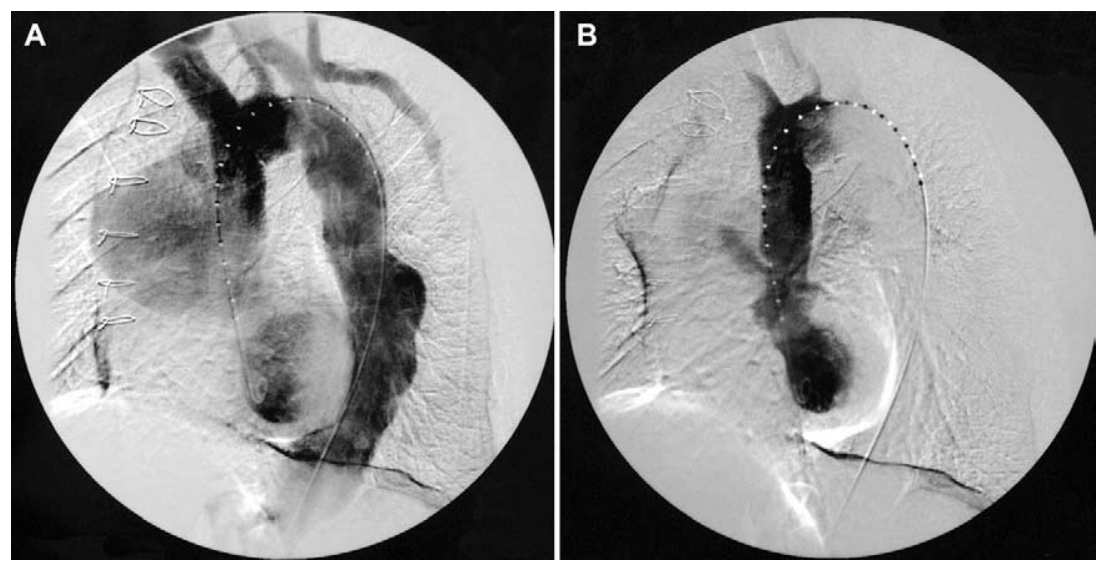

Figure 1. Angiography depicting a giant pseudoaneurysm (A) and the origin of the pseudoaneurysm located at the distal anastomosis of the Freestyle root replacement (B).
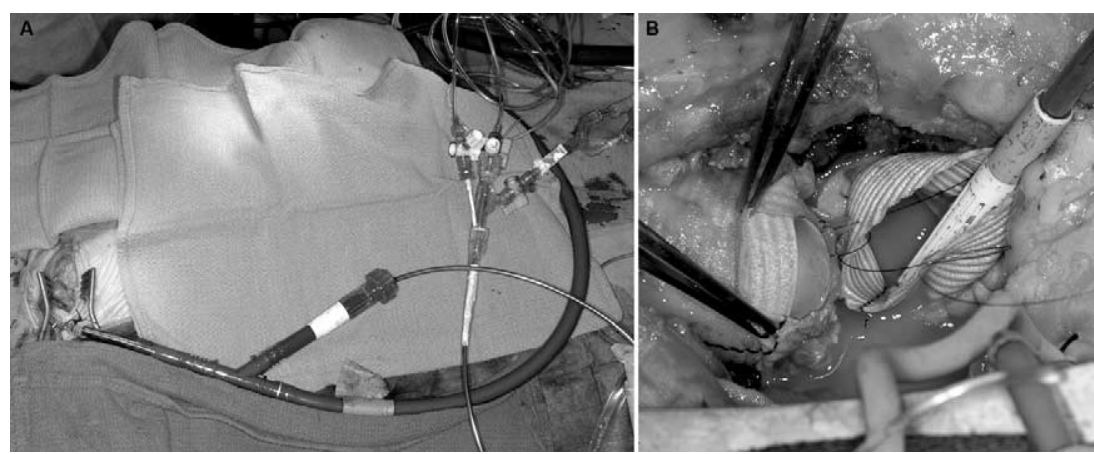

Figure 2. A, Redo axillary artery cannulation with an 8-mm Dacron graft and the use of a Y-branched arterial cannula, allowing insertion of the Endoclamp device. B, Operative view showing the Endoclamp device inflated in the ascending aortic graft and completion of the interposition graft.

thus minimizing the risks of embolic complications compared with a retrograde femoral perfusion, and also facilitates initiation of selective antegrade cerebral perfusion if circulatory arrest becomes necessary. A meticulous dissection and minimal cautery use reduce neurovascular damage related to the redissection of the axillary artery. Once CPB is initiated, the $10.5 \mathrm{~F}$ Endoclamp device is inserted in the side branch of the arterial cannula and positioned in the ascending aortic graft under fluoroscopy and transesophageal guidance. Monitoring of both radial pressures is important to assess the presence of flow obstruction from the Endoclamp device. In such circumstances, the right radial pressure will be significantly higher because of preferential perfusion. Snaring of the right axillary artery distal to the 8 - $\mathrm{mm}$ graft ensures adequate systemic perfusion. In the advent of high perfusion pressures, addition of a second arterial line in the common femoral artery or the left axillary artery is required. Once CPB flow is satisfactory, the Endoclamp device is inflated, the heart is arrested, and the sternum is reopened. In the presence of significant aortic regurgitation, a percutaneous retrograde cardioplegia catheter (Cardiovations) is placed in the coronary sinus.

In summary, we report a new technique to treat a giant pseudoaneurysm of the ascending aorta by means of the Endo-CPB platform and the reuse of the right axillary artery for cannulation. A prerequisite for this technique is the presence of an adequate site for the Endoclamp device inflation, preferably a Dacron graft, as in the present case. Moreover, origin of the pseudoaneurysm must be well documented and be localized proximally to the site at which the Endoclamp device will be inflated. This technique obviates the use of profound hypothermia, thus reducing CPB duration and its complications.

\section{References}

1. Schepens MA, Dossche KM, Morshuis WJ. Reoperations on the ascending aorta and aortic root: pitfalls and results in 134 patients. Ann Thorac Surg. 1999;68:1676-80.

2. Dossche KM, Tan ME, Schepens MA, et al. Twenty-four year experience with reoperations after ascending aortic or root replacement. Eur J Cardiothorac Surg. 1999;16:607-12.

3. Dougenis D, Daily BB, Kouchoukos NT. Reoperations on the aortic root and ascending aorta. Ann Thorac Surg. 1997;64:986-92.

4. Estrera AL, Miller CC III, Porat E et al. Determinants of early and late outcome for reoperations of the proximal aorta. Ann Thorac Surg. 2004;78:837-45. 